Check for updates

Cite this: RSC Adv., 2019, 9, 19740

Received 4th May 2019

Accepted 24th May 2019

DOI: $10.1039 / c 9 r a 03323 h$

rsc.li/rsc-advances

\section{Preparation and antimicrobial activity of thyme essential oil microcapsules prepared with gum arabic $\dagger$}

\begin{abstract}
Chenchen Cai, (D) Ruijia Ma, Mengwen Duan and Dengjun Lu*
Thyme essential oils (TEO) exhibit antimicrobial activities against a wide range of pathogenic microorganisms. Microcapsulation technology can be used to improve the stability, water solubility and antibacterial performance of TEO. In this paper, TEO was selected as the core material, and $\beta$-cyclodextrin ( $\beta$-CD) was the wall material for microcapsulation; gum arabic (GA) was used as an emulsifier to prepare microcapsules by coprecipitation. The effects of gum arabic on the encapsulation rate, particle size and release rate of microcapsules were investigated. The optimal condition was found to be TEO : GA by $1: 3(\mathrm{w} / \mathrm{w})$ ratio. In this condition, the embedding rate, release rate, and average size of the microcapsules were $87.61 \%, 53.00 \%$, and $8.20 \mu \mathrm{m}$, respectively. Scanning electron microscopy (SEM) revealed that, under the action of gum arabic, the surface of microcapsules was more complete, and the size apparently decreased. Fourier-transform infrared spectroscopy (FTIR) indicated that there was no significant chemical interaction between gum arabic and $\beta$ $\mathrm{CD}$. Gum arabic acted only as an emulsifier and remained in the mixed solution. For microcapsules with gum arabic as an emulsifier, the cumulative release rate of essential oils were slower at the initial time compared to microcapsules without added gum arabic. Antimicrobial activity assay exhibited TEO, which showed an inhibitory effect against Botryodiplodia theobromae Pat., and the inhibitory effect was especially strong against Colletotrichum gloeosporioides Penz. Finally, the obtained microcapsules showed the same antibacterial effect.
\end{abstract}

\section{Introduction}

The demand for a safe and natural food preservative has been rising currently in the market. Increasing concern over the side effects of chemical fungicides have enhanced the need to find antimicrobial molecules in nature. Natural antibacterial agents are gaining increasing attention from researchers and the industry due to their potential to provide quality and safety, as well as extend the shelf life of food products..$^{1-4}$ For example, essential oils (EOS) have received extensive attention in food preservation due to their effective combined activity against various bacteria and fungi.. ${ }^{5-7}$ Antimicrobial properties of essential oils from various plant species have been proven to affect and arrest microbial development.

Numerous in vitro studies have demonstrated the activity of different essential oils against bacteria, mold and yeast..$^{8-10}$ There is an important relationship between the chemical composition of essential oils and their antibacterial activity. These chemicals are biodegradable to nontoxic products and are potentially suitable for integrated use. The natural origin of essential oils (EO), in conjunction with their antimicrobial and antioxidant activity,

College of Light Industry and Food Engineering, Guangxi University, Nanning 530004, China.E-mail: dj6688@gxu.edu.cn

$\dagger$ Electronic supplementary information (ESI) available. See DOI: 10.1039/c9ra03323h makes them attractive for use in the food industry. Nevertheless, the direct addition of EO to the foods matrix has several disadvantages, such as rapid loss by volatilization and activity loss due to the effects of ultraviolet light and oxygen. ${ }^{11}$ However, an essential oil releasing rapidly may affect antimicrobial properties. To overcome the high volatility of essential oil components, encapsulation has emerged as a new alternative. ${ }^{12}$

The main components of thyme essential oils (TEO) include thymol, carvacrol and paracymene..$^{13,14}$ Thyme essential oil has been found to have antibacterial and antifungal effects on various pathogenic microorganisms. ${ }^{15,16}$ Thyme essential oil highly reduced $64.00 \%$ of gray mold incidence in tomato plants and reduced Fusarium wilt severity to $30.76 \%$ in tomato plants grown in the hydroponic system. ${ }^{17}$

As a wall material for microcapsules, cyclodextrin (CD) has been studied extensively. Cyclodextrins are toroidal-shaped cyclic oligosaccharides with a hydrophilic outer surface and an internal hydrophobic hollow interior, which can entrap a vast number of lipophilic compounds into their hydrophobic cavity, depending on their size and molecular structure. ${ }^{18}$ The most common CDs are formed from six $(\alpha-\mathrm{CD})$, seven ( $\beta$-CD) or eight $\left(\gamma\right.$-CD) $\mathrm{D}$-glucopyranose units. ${ }^{19}$ The three major CDs are crystalline, homogeneous, and nonhygroscopic substances, which are torus-like macro-rings that are built up from glucopyranose units. ${ }^{20} \beta$-Cyclodextrin ( $\beta$-CD) is a cyclic oligosaccharide that is composed of $7 \mathrm{D}(+)$-glucopyranoses, which have 
a hydrophobic inner cavity and can be used with a wide range of objects. ${ }^{21}$ Among the various wall materials, $\beta$-CD is one of the most effective and simple microcapsule systems and is used widely in the encapsulation process to form an inclusion complex. Encapsulation in $\beta$-CD is one of the most effective methods for protecting active compounds against oxidation, heat degradation, and evaporation, as well as for masking undesired scents or flavors and increasing solubility. ${ }^{22}$

As part of the method for preparing microcapsules, the saturated aqueous solution method is also called a coprecipitation method, ${ }^{23,24}$ in which a cyclodextrin is made into a saturated aqueous solution, and a guest molecule is added. For those insoluble molecules in water, a small amount of a suitable solvent (such as ethanol) may be added. After dissolution, the mixture is stirred and mixed for a certain period of time to allow the guest molecules to be encapsulated. Then, the mixed liquid is subjected to low-temperature refrigerating precipitation and filtration, and then is washed and dried according to the nature of the guest molecule, with a suitable solvent to obtain a stable clathrate. ${ }^{25,26}$ In addition, several researchs have reported the use of polylactide, for the encapsulation of TEO. ${ }^{27-29}$

Gum arabic (GA) is one of the popular ingredients that is widely used in the food and pharmaceutical industries. Gum arabic is a natural acacia tree exudate that contains hyperbranched polysaccharides and proteins. ${ }^{30}$ Fractions are mainly composed of arabinogalactan (90\% of total mass), arabinogalactan protein ( $10 \%$ of total mass) and glycoprotein ( $1 \%$ of total mass). ${ }^{31,32}$ GA is commonly used as an emulsifier to stabilize emulsions, the emulsifying property of which is provided by an excellent interfacial property of arabinogalactan protein. The structure of arabinogalactan protein provides both hydrophobic polypeptide chain and hydrophilic carbohydrate blocks, which confers good emulsification characteristics. ${ }^{33}$ The function of GA is provided by repulsive electrostatic and steric interactions after the polypeptide moieties adsorb onto the oil droplet surface and the polysaccharide chains protrude in the aqueous phase. ${ }^{34}$ The high water solubility and low solution viscosity increase the possibility of embedding essential oils.

In the present study, microcapsules based on $\beta$-cyclodextrin, thyme essential oil, and gum arabic were produced to investigate the effect of the inclusion rate of essential oil. And gum arabic was, for the first time, used as an emulsifier for microcapsulation of essential oil. This study analyzed the antimicrobial effects of thyme essential oil and microcapsules for future applications as an antibacterial agent in food commodities. The gum arabic as an emulsifier exhibited excellent effect on embedding rate and release rate.

\section{Material and methods}

\section{Materials}

All chemicals and solvents were of analytical grade. $\beta$-Cyclodextrin ( $\beta$-CDs), gum arabic (GA) and thyme essential oil (TEO, $99 \%$ ) were supplied by Yuanye, China. Deionized water was used throughout the synthesis and analysis.

Colletotrichum gloeosporioides Penz. and Botryodiplodia theobromae Pat. were obtained and isolated from infected mangos in the laboratory of the Guangxi Academy of Agricultural Sciences (Nanning, China).

\section{Production of thyme essential oil microcapsules}

The microcapsules were prepared according to the optimized formulation and fabrication method described by. ${ }^{35,36} \beta-\mathrm{CD}(2.5 \%$ $\mathrm{w} / \mathrm{v}$ ) and GA (with the ratio of TEO : GA = $1: 0,1: 1,1: 3,1: 5$, $1: 7,1: 9, \mathrm{w} / \mathrm{w})$ were prepared by dispersing deionized water and were stirred with $600 \mathrm{rpm}$ for $2 \mathrm{~h}$ at $60{ }^{\circ} \mathrm{C}$ to obtain a homogeneous mixture. This oil phase was then mixed with $40{ }^{\circ} \mathrm{C}$ preheated anhydrous ethanol to obtain a ratio of $1 \%(\mathrm{w} / \mathrm{v})$. TEO was then added dropwise into the $\beta$-CDs/GA mixture under gentle stirring at $40{ }^{\circ} \mathrm{C}$ and $1000 \mathrm{rpm}$. The suspension was obtained after stirring $6 \mathrm{~h}$. This mixture was cooled to room temperature. To accelerate precipitation, the mixture was left at $4{ }^{\circ} \mathrm{C}$ overnight. The precipitated TEO/ $\beta$-CD complex was acquired by vacuumfiltration using $0.22 \mu \mathrm{m}$ microfiltration membranes, and the precipitate was washed with ethanol three times and was heated in a vacuum oven at $50{ }^{\circ} \mathrm{C}$ until constant weight. The collected dry powder was named TEO/ $\beta$-CD microcapsules.

\section{Ultraviolet-visible (UV-vis)}

Ultraviolet-visible (UV-vis) absorption spectra of prepared microcapsules supernatant was recorded using a UV-vis spectrophotometer (UV-4100, Shimadzu Corporation, Tokyo, Japan) over wavelengths ranging from 200 to $500 \mathrm{~nm}$. The maximum wavelength absorption is $293 \mathrm{~nm}$, which is close to the absorption wavelength that was reported elsewhere. ${ }^{37}$

\section{Microencapsulation process yield ( $Y \%)$}

The yield is the rate between the mass of microcapsules obtained and the initial mass of $\beta-C D$ and TEO. The sample concentration was calculated using the calibration curve. For the construction of the calibration curve, TEO was used at different known concentrations. The microencapsulation process yield was calculated by using the following expression:

$$
Y(\%)=\frac{M_{\mathrm{mc}}}{M_{\mathrm{cd}}+M_{\mathrm{o}}} \times 100
$$

where $M_{\mathrm{mc}}, M_{\mathrm{cd}}$ and $M_{0}$ are the mass of microcapsules obtained, the initial weight of $\beta-\mathrm{CD}$ and the initial weight of TEO, respectively.

\section{Assay of thyme essential oil embedding rate (EE\%)}

The amount of TEO encapsulated in $\beta$-CD was determined by a UV-visible spectrophotometer following the method described by, ${ }^{38,39}$ with few modifications. Briefly, a $10 \mathrm{mg}$ sample was poured into ethanol and soaked overnight. The supernatant was centrifuged for $10 \mathrm{~min}$ at $12000 \mathrm{rpm}$. Absorbance of the obtained solution was measured, and ethanol was used as a reference. Three groups were made in parallel, and the average value was calculated. The sample concentration was calculated using the calibration curve. The $\mathrm{EE} \%$ of the microencapsulation process were calculated by using the following expressions: 


$$
\mathrm{EE} \%=\frac{M_{\mathrm{eo}}}{M_{\mathrm{o}}} \times 100
$$

where $M_{\text {eo }}$ is the weight of TEO encapsulated.

\section{Assay of thyme essential oil release rate}

Thyme essential oil released from the microcapsulations were evaluated. In vitro release of thyme essential oil from $\beta$-cyclodextrin in phosphate buffers was carried out according to reported methods, ${ }^{\mathbf{4 0 , 4 1}}$ with slight modifications. Briefly, a certain amount of microencapsulation and buffer solution $(10 \mathrm{~mL})$ in dialysis membrane bags (8000-14 000 MWCO, width $77 \mathrm{~mm}$ ) were placed into $100 \mathrm{~mL}$ phosphate buffers at $25{ }^{\circ} \mathrm{C}$ with constant stirring. Then, $4 \mathrm{~mL}$ of phosphate buffer was withdrawn for analysis and was replaced with an equivalent volume of fresh buffer to maintain the total volume. Aliquots of $4 \mathrm{~mL}$ were removed every $2 \mathrm{~h}$, and the initial volume $(100 \mathrm{~mL})$ was maintained by the addition of phosphate buffers. The release of thymol essential oil was monitored by UV-vis absorption spectroscopy. The cumulative release percentage of TEO was determined by using the following equation. The analysis of each sample was performed in triplicate.

$$
\text { Cumulative release percentage }=\sum_{t=0}^{t} \frac{M_{t}}{M_{0}} \times 100
$$

where $M_{t}$ and $M_{0}$ are the cumulative amount of TEO at each sampling time and the initial weight of the TEO-loaded in the sample, respectively.

\section{Particle-size analyses}

Measurement of mean particle hydrodiameter of the microcapsulations were determined by the size and zeta potential analyzer (Zetasizer Nano ZSP, UK). Data were reported as the averages of the three readings.

\section{Scanning electron microscopy (SEM)}

The surface micrographs of the TEO microcapsule particles were imaged by scanning electron microscopy (F16502, Phenom, Netherlands). SEM images provided the morphology and approximate size of the crystals that formed by the inclusion complexes. Microcapsules were attached to the specimen holder with a double-sided adhesive tape and were sputtered with gold. SEM images were taken at the required magnification at room temperature and examined using an acceleration voltage of $25 \mathrm{kV}$.

\section{Fourier transform infrared spectroscopy (FTIR)}

To confirm the information about chemical bond or functional group, the FTIR spectra of the samples were obtained by using a BRUKER TENSOR II instrument (Bruker, Germany). Approximately $1 \mathrm{mg}$ of sample was mixed with approximately $100 \mathrm{mg}$ potassium bromide $(\mathrm{KBr})$ and was pressurized to achieve a transparent pallet. Each spectrum was recorded in a frequency range of $800-4000 \mathrm{~cm}^{-1}$. Each sample was scanned 16 times with a resolution of $4 \mathrm{~cm}^{-1}$.

\section{Antimicrobial activity}

The antimicrobial activity was evaluated by the agar diffusion assay. Colletotrichum gloeosporioides Penz. and Botryodiplodia theobromae Pat. were used as the test microorganisms. The samples' antimicrobial activity was evaluated by the modified agar diffusion method. The microbial strains were cultured on dextrose agar for fungi and were incubated at $28 \pm 2{ }^{\circ} \mathrm{C}$ for $48 \mathrm{~h}$ for fungi. The antimicrobial activity of the thyme essential oil and microcapsules were determined.

The different concentrations of TEO $(0.0,10.0,125.0,166.6$, 250.0 and $500.0 \mu \mathrm{L} \mathrm{mL} \mathrm{m}^{-1}$ ) were prepared in an appropriate solvent (dimethyl sulfoxide). The different TEO concentrations were sterilized by a $0.45 \mu \mathrm{m}$ syringe microfilter. For this purpose, $20 \mathrm{~mL}$ inoculated medium was added to each Petri dish (90 $\mathrm{mm} \times 15 \mathrm{~mm}$ ) and was allowed to solidify (dextrose agar for fungi).

For the antibacterial activity of thyme essential oil, $7 \mathrm{~mm}$ diameter filter papers mixed with thyme essential oils lime at concentrations of $0.0,10.0,125.0,166.6,250.0$ and $500.0(\mathrm{v} / \mathrm{v})$ were placed in the center of the culture dish cover. A PDA agar disk (7 mm diameter) of a pure culture of Colletotrichum gloeosporioides Penz. and Botryodiplodia theobromae Pat. was placed onto the center of a PDA plate, respectively. Plates were incubated at ambient temperature $\left(28 \pm 2{ }^{\circ} \mathrm{C}\right)$.

For the antibacterial activity of microcapsules, the PDA plate was mixed with the different weights of thyme essential oils microcapsules $(0.2,0.4,0.8 \mathrm{~g})$. A PDA agar disk ( $7 \mathrm{~mm}$ diameter) of a pure culture of fungi was placed onto the center of a PDA plate. The antimicrobial activity of microcapsules was evaluated by the diameter of the fungi agar disk.

At the same time, the antibacterial effect of $\beta$-cyclodextrin and gum arabic was also evaluated. The diameters were measured to the nearest $0.02 \mathrm{~mm}$. Daily radial growth measurements were taken until the control dishes were fully covered with mycelia. Three plates for each treatment were considered for this evaluation, and the results were analyzed statistically. The average of diameters for each sample were used to determine the antimicrobial activity. At the same time, the antibacterial activity of each sample were also observed further under the same experimental conditions.

\section{Statistical analysis}

The results were treated statistically using SPSS software (Version 20), and data were expressed as the means \pm standard deviations (S.D.). Differences were regarded as significant when $p<0.05$.

\section{Results and discussions}

TEO microcapsules were prepared by coprecipitation technology. In this process, the effects of GA on the morphology and embedding rate of microcapsules were investigated.

\section{The calibration curve for thyme essential oil}

Loading of TEO into the $\beta$-CD and its release was monitored by UV-vis absorption spectroscopy. The amounts of TEO encapsulated in $\beta$-cyclodextrin were determined using a calibration 
Table 1 Effect of TEO : GA weighted ratio on the physicochemical properties of TEO-loaded $\beta-C D$. The results are reported in the mean $\pm \mathrm{SD}, n=3^{a}$

\begin{tabular}{lllr}
\hline $\begin{array}{l}\text { TEO : GA } \\
(\mathrm{w} / \mathrm{w})\end{array}$ & $\begin{array}{l}\text { Yields of } \\
\text { microcapsules }(Y \%)\end{array}$ & $\begin{array}{l}\text { Encapsulation } \\
\text { efficiency }(\mathrm{EE} \%)\end{array}$ & \multicolumn{1}{l}{$\begin{array}{l}\text { Average } \\
\text { diameter }(\mu \mathrm{m})\end{array}$} \\
\hline $1: 0$ & $75.93 \pm 3.43$ & $38.87 \pm 3.40$ & $10.50 \pm 1.40$ \\
$1: 1$ & $78.60 \pm 2.57$ & $40.24 \pm 1.32$ & $9.70 \pm 2.10$ \\
$1: 3$ & $87.61 \pm 1.13$ & $53.00 \pm 1.12$ & $8.20 \pm 1.67$ \\
$1: 5$ & $81.81 \pm 1.64$ & $41.23 \pm 2.37$ & $9.78 \pm 1.07$ \\
$1: 7$ & $85.63 \pm 1.34$ & $40.07 \pm 3.02$ & $9.98 \pm 2.01$ \\
$1: 9$ & $78.58 \pm 3.67$ & $37.72 \pm 3.86$ & $10.67 \pm 2.77$
\end{tabular}

${ }^{a} Y=$ (amount of microcapsules/initial amount of TEO and $\beta$-CD) $\times 100$, $\mathrm{EE}=($ amount of TEO loaded/initial amount of TEO $) \times 100$.

curve that related the ultraviolet absorbance values with the thyme essential oil concentration values in an ethanol solution. The TEO exhibited a maximum wavelength absorption at $293 \mathrm{~nm}$. A linear correlation was obtained between the magnitude of the absorbance peak and the concentrations of thyme essential oil in the ethanol solutions, which permitted the establishment of a calibration curve. This linear correlation was defined as the calibration curve for essential oil and was utilized to determine the essential oil concentrations in the $\beta$-cyclodextrin structures after the encapsulation processes.

\section{Microencapsulation process yield (Y\%)}

With the weighted ratio of gum arabic and essential oil increased, the percentage yield of the essential oil microcapsule varied within a certain range. When the weighted ratio of essential oil was $1: 3$, the maximum percentage yield of the essential oil microcapsule was $87.61 \%$ (Table 1 ), which may be due to the increase in weight of GA that was caused by the change in the solubility of cyclodextrin in water.

\section{Encapsulation efficiency of thyme essential oil}

The encapsulation efficiency (\%EE) of different weighted data are shown in Table 1 . The result shows that \%EE increases and then decreases with increasing GA content. The presence of GA (a)

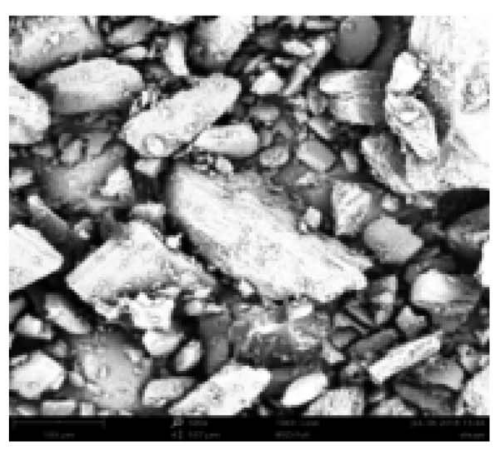

(c)

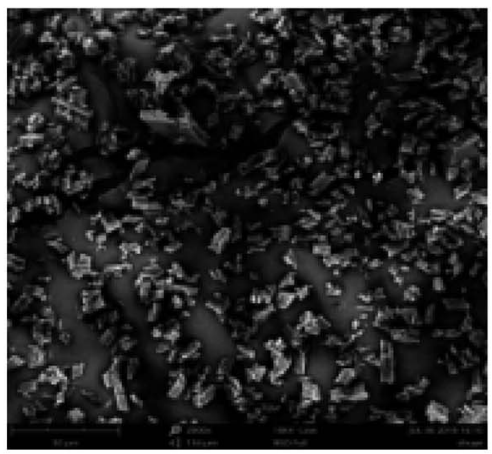

(e)

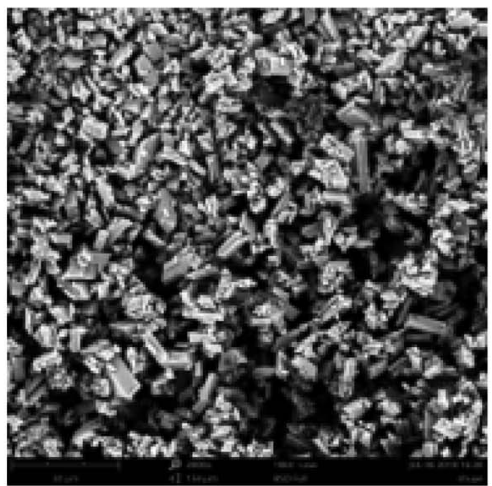

(b)

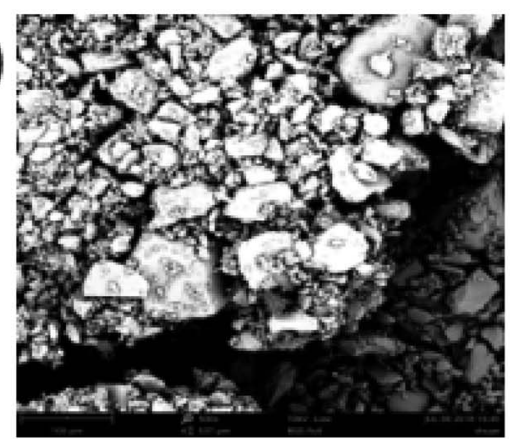

(d)

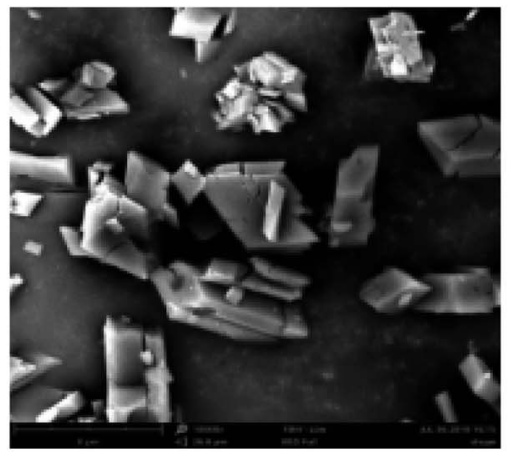

(f)

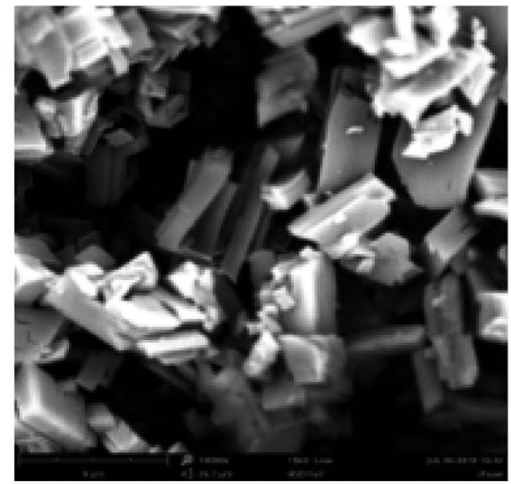

Fig. 1 SEM micrographs of $\beta-C D(a)$, the GA (b), the $\beta$-CD/TEO microcapsules (c and d) and, $\beta$-CD/GA-TEO microcapsules (e and f). (c and e: magnified 2000 times; $d$ and f: magnified 10000 times). 


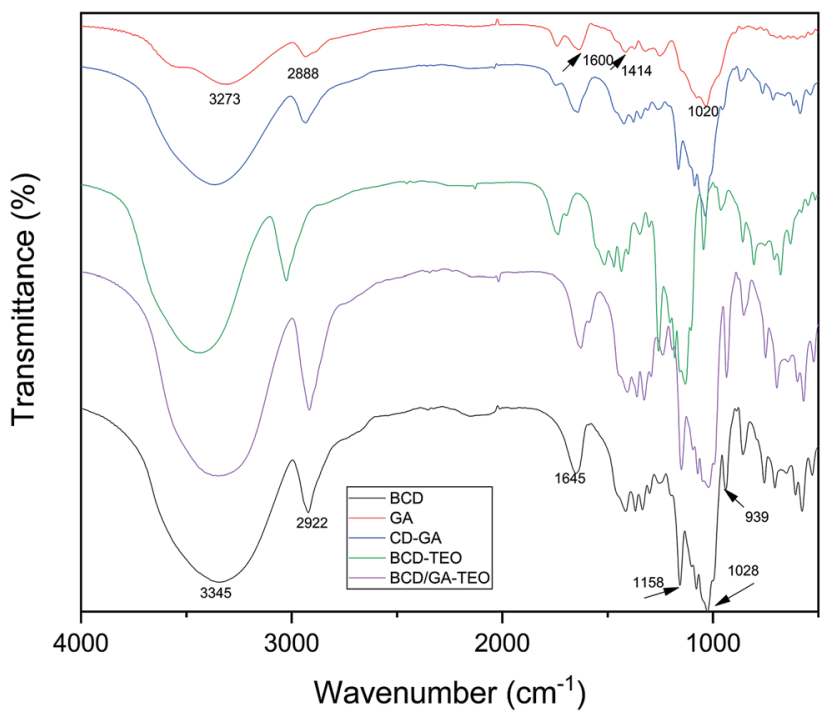

Fig. 2 FTIR spectra of gum arabic (GA), physical mixing of gum arabic with $\beta$-cyclodextrin (CD-GA), thyme essential oil/ $\beta$-cyclodextrin complexes (BCD-TEO), and thyme essential oil/ $\beta$-cyclodextrin complexes using gum arabic as an emulsifier (BCD/GA-TEO). Additionally, FTIR spectra for pure $\beta$-cyclodextrin (BCD) is shown.

increased the embedding rate of TEO. When the weighted ratio of essential oil was $1: 3$, the maximum embedding rate was $53.00 \%$. The emulsion droplets of TEO probably moved more freely in the medium. ${ }^{42}$ Due to the increased amount of gum arabic, the flow of essential oil was hindered. At the appropriate emulsifier concentration, the rate at which the essential oil molecules diffused at the mixed solution was probably higher. At the same time, this could provide a better protection of oil from coalescence which ultimately embeds in the $\beta$ cyclodextrin.

\section{The mean diameter of the microcapsules}

The microcapsules were characterized and compared in terms of the mean particle size. The optimum dosage of gum arabic was determined. Significant difference was found in the size of the microcapsules prepared with the different weighted ratio of the TEO : GA. The mean diameter of TEO loaded microcapsules decreased appreciably as the TEO : GA weighted ratio increased from $1: 0$ to $1: 3$, followed by slight increase when the TEO : GA weighted ratio increased from $1: 3$ to $1: 9$ (Table 1 ). When the weighted ratio of the TEO : GA was $1: 3$, the minimum particle size of microcapsules was obtained. The presence of GA affected the microcapsules size. GA includes approximately $2 \%$ protein and can form stable emulsions with most oils. ${ }^{43}$ Therefore, the size of the finally obtained microcapsules became dependent upon the effect of gum arabic concentration during the preparation process.

\section{Scanning electron microscopy (SEM)}

The relatively hydrophobic cavity of $\beta$-CD can accommodate various kinds of guest molecules to form inclusion complexes with different morphologies. The SEM morphology with images of $\beta$-CD/TEO is illustrated in Fig. 1(c-f). Obviously, some microcapsules had the typical monoclinic crystalline structures surface, ${ }^{44}$ which was a unique feature of $\beta$-CD microcapsules prepared by the coprecipitation method. Most microcapsules had a relatively smooth, intact surface.

As shown in Fig. 1(a and b) the pure sample of $\beta-\mathrm{CD}$ and GA exists in amorphous, irregular crystal and varied sizes without a defined shape. The average particle sizes were $88.88 \mu \mathrm{m}$ for $\beta$ $\mathrm{CD}$ and $67.08 \mu \mathrm{m}$ for GA (Fig. 1a and b). Fig. 1(c-f) demonstrated the typical morphology of the $\beta$-CD/TEO complexes and the $\beta$-CD/GA-TEO complexes together with the diameter distribution of sample. The $\beta$-CD/TEO complexes showed the typical monoclinic crystalline structures that have been reported for the inclusion of thymol in this type of $\beta$-CD. ${ }^{45}$ The average size varied between 8 and $10 \mu \mathrm{m}$ (Fig. 1c). The microcapsules with gum arabic showed the typical monoclinic crystalline structures where the average size was smaller than the average particle sizes of $\beta$-CD/TEO complexes without GA. Then, the relationship between the particle size of microcapsules and the particle release rate was also found. The microcapsule with smaller particles had slower release rate than those having larger size. This finding corresponds with the result that was obtained from the study on the release rate, as discussed later. ${ }^{46}$ Munhuweyi et al. studied the complexes of $\beta$-CD and EOs that were obtained by the coprecipitation method and reported crystal complexes with smaller rhomboidal crystals. In the presence of gum arabic, the overall density of microcapsules increased, and the surface was smoother, which indicated levels of breakage. With $\beta$-CD as its wall materials and GA as an emulsifier, the TEO microcapsule displayed a surface morphology and good particle size distribution.

Specific types of complexation may be demonstrated by the FTIR spectra where some isolated absorption band was correlated with the complexation process. Guest molecules can alter the intensity and relative location of the bands. FTIR spectroscopy was used to evaluate the effects of gum arabic on microcapsules. FTIR spectra of different samples are shown in Fig. 2.

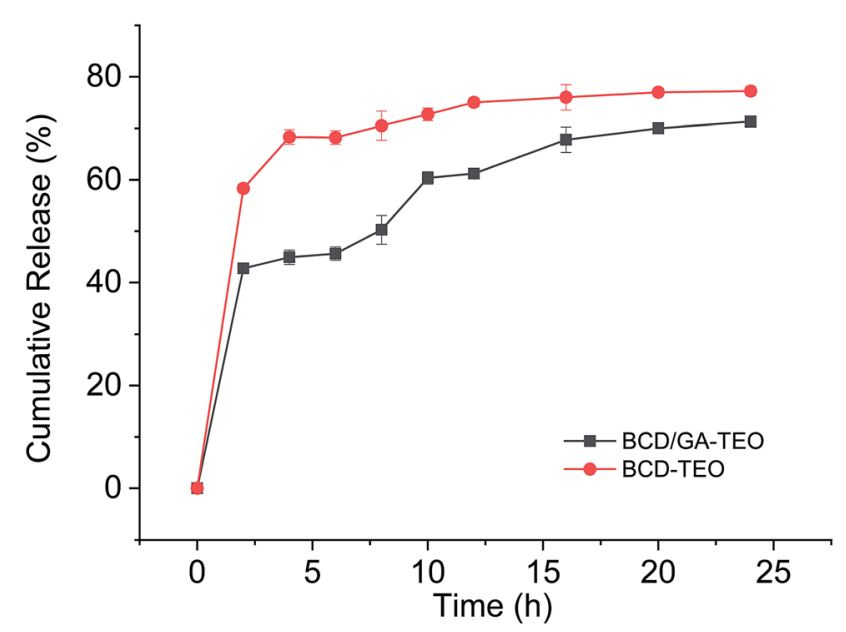

Fig. 3 In vitro release profiles of CEO from thyme essential oil/ $\beta$ cyclodextrin complexes (BCD-TEO) and thyme essential oil/ $\beta$ cyclodextrin complexes using gum arabic as an emulsifier (BCD/GATEO). Data were represented as the mean $\pm \mathrm{SD}, n=3$. 
The spectrum of $\beta$-CD showed absorption bands at $3345 \mathrm{~cm}^{-1}$ (symmetrical and asymmetrical stretching of the -OH groups), $2922 \mathrm{~cm}^{-1}$ (C-H stretching), $1645 \mathrm{~cm}^{-1}$ (O-H bending), $1154 \mathrm{~cm}^{-1}$ and $1026 \mathrm{~cm}^{-1}$ (asymmetric and symmetric stretching of the $\mathrm{C}-\mathrm{O}-\mathrm{C}$; Fig. 2). Comparable studies have been reported. ${ }^{47}$

Gum arabic presented strong vibrational peaks at $1020 \mathrm{~cm}^{-1}$ (C-O stretching), $1414 \mathrm{~cm}^{-1}$ ( $\mathrm{CH}_{3}$ bending and $\mathrm{C}-\mathrm{H}$ bending), $1600 \mathrm{~cm}^{-1}$ ( $\mathrm{C}=\mathrm{O}$ stretching) and $2888 \mathrm{~cm}^{-1}$ (low intensity of a carboxylic group). The band that presented at $3273 \mathrm{~cm}^{-1}$ indicates the presence of hydrogen-bonded $\mathrm{OH}$ group. ${ }^{48}$ The characteristic absorption band in the region of $3400-3500 \mathrm{~cm}^{-1}$ for the amino group of gum arabic must have been masked by the broad $\mathrm{O}-\mathrm{H}$ group absorption band. ${ }^{49}$

Small differences in the spectra of BCD/GA-TEO and BCDTEO complexes were observed, presumably due to the presence of small quantities of gum arabic. According to the physicochemical tests performed, we demonstrated with evidence that the GA was only used as an emulsifier and was not used as an ingredient in the microcapsules.

\section{Release studies}

The release behaviors of microcapsule in the dissolution media are been presented in Fig. 3 . The release of TEO from complexes can be divided into two phases. For the first phase (the first $5 \mathrm{~h}$ ), the mechanism of release is attributed to the release of molecules that have the weaker affinity with $\beta$-cyclodextrin. The second phase is slow-release where the rate of release decreases slightly. The mechanism of release is attributed to the high stability between $\beta$-CD and carvacrol, thymol. ${ }^{50,51}$ These results can also explain the antibacterial activity.

In BCD-TEO complexes, a higher oil release profile was observed than that prepared with $\mathrm{BCD} / \mathrm{GA}-\mathrm{TEO}$ complexes. The microcapsules with GA in the preparation process, in which the release rate of essential oil increased with time and then tended to be stable. For the control group that has microcapsules without GA in the preparation process, the release rate of essential oil increased rapidly in the initial time. First, the release behaviors of the microcapsules increase with the increase of particle size microcapsules. The physical connection between gum arabic and cyclodextrin resulted in a reduction in the gap between the microcapsule molecules, which impedes the release of essential oils. Only a fraction of the GA acted as barriers, and a large proportion of GA were found in the residual raw material liquid of the microcapsule. The effect of gum arabic on the release of essential oil needs to be studied further.

\section{Antibacterial activity}

An agar disk diffusion test was carried out to investigate the antibacterial activity of the thyme essential oil and microcapsule against fungi. Thyme essential oils have a certain antibacterial effect on Colletotrichum gloeosporioides Penz. and Botryodiplodia theobromae Pat., and their growth was reduced as the essential oil concentration increased. When the concentration of thyme essential oil was up to $500\left(\mu \mathrm{L} \mathrm{mL}^{-1}\right)$, a slight rate of growth was observed in both fungi (Fig. 4a). According to previous reports, the antibacterial activity of essential oils may be due to the ability of essential oils to penetrate cell membranes, destroy cell membranes, inhibit cell functional properties, and, ultimately, lead to cell contents leakage leading to cell death.

In Fig. 4(a and b), the diameters (mm) of the PDA agar disk of a pure culture of fungi are shown. The diameter $(\mathrm{mm})$ values showed that the antibacterial effects of the sample on Colletotrichum gloeosporioides Penz. were better than that of Botryodiplodia theobromae Pat., for both the thyme essential oil and an essential oil microcapsule. The antimicrobial effects of thyme essential oil on Colletotrichum gloeosporioides Penz. have been reported. ${ }^{22,53}$ The research of agar diffusion tests obtained for the $\beta$-cyclodextrin molecules and GA indicated that the $\beta$-CD and GA molecules did not have antimicrobial properties.

In Fig. 5(a and b), during the 7 day culture period, the essential oil was released gradually from the hydrophobic cavity to the surface of $\beta$-CD. Over time, this process could weaken the
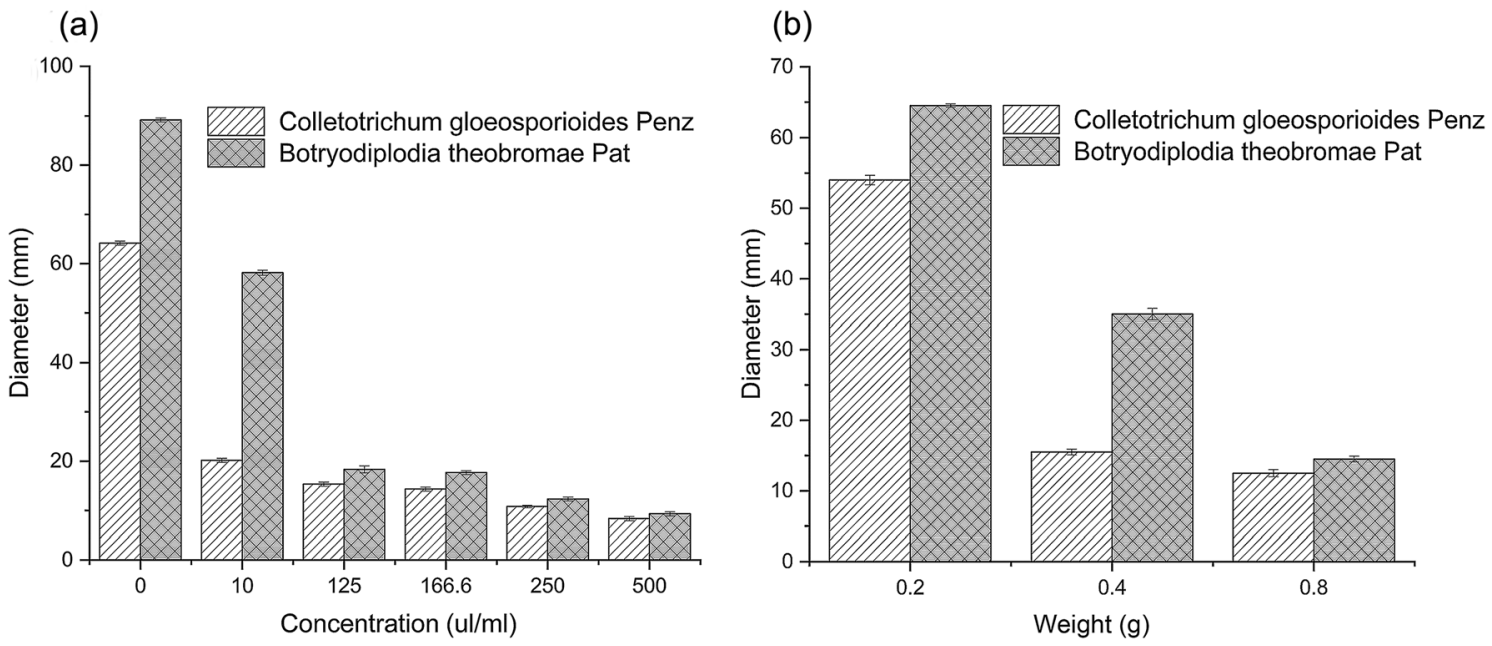

Fig. 4 The antibacterial activity (a) thyme essential oils and (b) thyme essential oil microcapsules on Colletotrichum gloeosporioides Penz. and Botryodiplodia theobromae Pat. Data were given as the means \pm S.D $(n=3)$. 
(a)

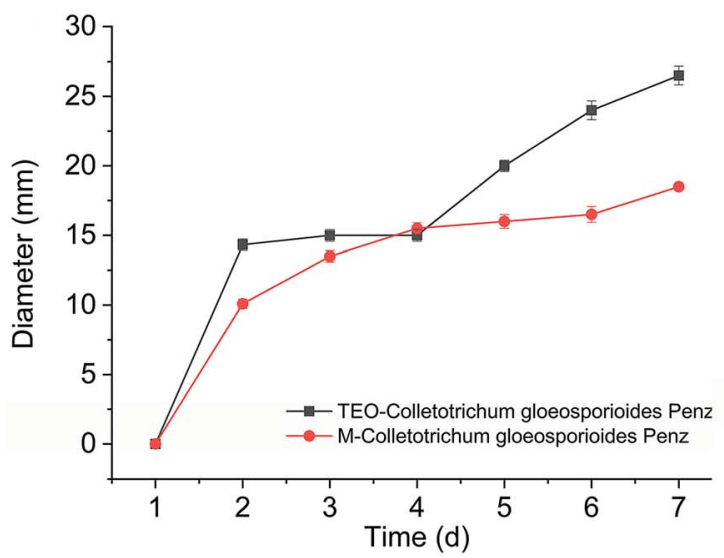

(b)

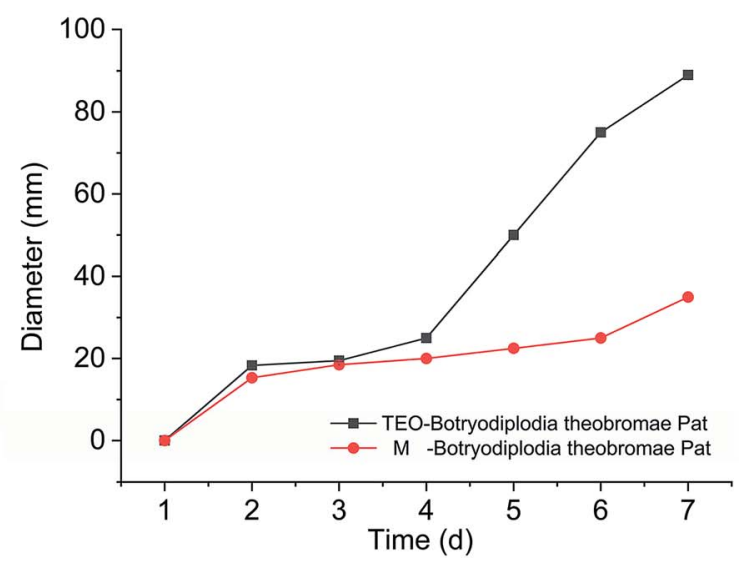

Fig. 5 The antibacterial activity of thyme essential oils and thyme essential oil microcapsules on (a) Colletotrichum gloeosporioides Penz., and (b) Botryodiplodia theobromae Pat. Data are given as the means \pm S.D $(n=3)$.

antibacterial effect, and the growth rate of the fungus began to accelerate. For the essential oil microcapsules, in the first 2 days, due to its slow release rate, it did not show an improved antibacterial effect; however, with the passage of time, its release rate was accelerated to a certain extent, which inhibited the growth of fungi very well. The microcapsules exhibited antibacterial effect over a certain period of time.

In this study, thyme essential oil showed good antibacterial effect on Colletotrichum gloeosporioides Penz. and Botryodiplodia theobromae Pat. Numerous studies have shown that Colletotrichum gloeosporioides Penz. and Botryodiplodia theobromae Pat., are the main strains that cause mango to accelerate the deterioration of mango after picking. In other studies, Amomum subulatum Roxb oil ${ }^{54}$ and mustard essential oil ${ }^{55}$ exhibited antifungal activity against two mango rotting fungi viz. including Colletotrichum gloeosporioides Penz. and Botryodiplodia theobromae Pat. However, these essential oils were not embedded in the shell material, and could not improve the controlled release of essential oil.

The two fungi in this experiment were obtained from mangos. The research results of this experiment might be the basis for the future application of thyme essential oil to mango preservation. However, thyme essential oil has not been studied for use as an additive to fruit preservation due to its strong odor, thermal instability and low flash point. ${ }^{56,57}$ These problems can be solved by embedding thyme essential oil with $\beta$-cyclodextrin.

\section{Conclusion}

Because gum arabic has good water solubility and emulsifying properties, it emulsifies in the process of preparing essential oil microcapsules, and the microencapsulation efficiency of the embedding material can be increased during the embedding process. The antibacterial effect showed that TEO and thyme essential oil microcapsule are capable of inhibiting Colletotrichum gloeosporioides Penz. and Botryodiplodia theobromae Pat. under proper growth conditions. The results of this study are beneficial for mango preservation. These findings indicate that the thyme essential oil microcapsule as a natural bacteriostatic agent that has the potential to be widely used in the food processing industry.

\section{Conflicts of interest}

The authors confirm that they have no conflicts of interest with respect to the work that is described in this manuscript.

\section{Acknowledgements}

The author(s) disclosed receipt of the following financial support for the research, authorship, and/or publication of this article. The authors received financial support from the Innovation Project of Guangxi Graduate Education (YCSW2019040).

\section{References}

1 A. Prakash, V. Vadivel, D. Rubini and P. Nithyanand, Food Biosci., 2019, 28, 57-65.

2 P. Farshi, M. Tabibiazar, M. Ghorbani, M. Mohammadifar, M. B. Amirkhiz and H. Hamishehkar, Food Biosci., 2019, 28, 49-56.

3 Z. Fang, L. Xu, Y. Lin, X. Cai and S. Wang, Food Control, 2019, 98, 24-33.

4 A. Valdés, A. C. Mellinas, M. Ramos, N. Burgos, A. Jiménez and M. C. Garrigós, RSC Adv., 2015, 5, 40324-40335.

5 D. Kalemba, M. Matla and A. Smętek, in Dietary Phytochemicals and Microbes, ed. A. K. Patra, Springer, Netherlands, Dordrecht, 2012, pp. 157-183.

6 J. Moura, F. Guerra, F. Pereira, J. Pereira De Sousa, V. Nogueira Trajano and E. de Oliveira Lima, Bol. Latinoam. Caribe Plant. Med. Aromat., 2012, 11, 208-217.

7 D. Chung, T. J. Cho and M. S. Rhee, Food Res. Int., 2018, 107, 578-588.

8 S. Burt, Int. J. Food Microbiol., 2004, 94, 223-253.

9 K. Svoboda, J. D. Brooker and J. Zrustova, Acta Hortic., 2006, 709, 35-43. 
10 P. An, X. Yang, J. Yu, J. Qi, X. Ren and Q. Kong, Food Control, 2019, 98, 42-53.

11 H. Majeed, Y. Bian, B. Ali, A. Jamil, U. Majeed, Q. F. Khan, K. J. Iqbal, C. F. Shoemaker and Z. Fang, $R S C A d v$., 2015, 5, 58449-58463.

12 M. Chen, Y. Hu, J. Zhou, Y. Xie, H. Wu, T. Yuan and Z. Yang, RSC Adv., 2016, 6, 13032-13039.

13 M. Mahboubi, R. Heidarytabar, E. Mahdizadeh and H. Hosseini, Agriculture and Natural Resources, 2017, 51, 395-401.

14 S. C. Purcell, P. Pande, Y. Lin, E. J. Rivera, L. Paw U, L. M. Smallwood, G. A. Kerstiens, L. B. Armstrong, M. T. Robak, A. M. Baranger and M. C. Douskey, J. Chem. Educ., 2016, 93, 1422-1427.

15 C. Vasile, M. Sivertsvik, A. C. Mitelut , M. A. Brebu, E. Stoleru, J. T. Rosnes, E. E. Tănase, W. Khan, D. Pamfil and C. P. Cornea, Materials, 2017, 10, 45.

16 C. M. Blanco-Lizarazo, R. Betancourt-Cortés, A. Lombana, K. Carrillo-Castro and I. Sotelo-Díaz, Food Sci. Technol. Int., 2016, 23, 277-288.

17 M. Ben-Jabeur, E. Ghabri, M. Myriam and W. Hamada, Plant Physiol. Biochem., 2015, 94, 35-40.

18 F. Kayaci, H. S. Sen, E. Durgun and T. Uyar, Food Res. Int., 2014, 62, 424-431.

19 V. J. Stella and Q. He, Toxicol. Pathol., 2008, 36, 30-42.

20 G. Astray, C. Gonzalez-Barreiro, J. C. Mejuto, R. Rial-Otero and J. Simal-Gándara, Food Hydrocolloids, 2009, 23, 16311640.

21 J. Wang and Z. Cai, Carbohydr. Polym., 2008, 72, 695-700.

22 A. Rukmani and M. Sundrarajan, J. Ind. Text., 2011, 42, 132144.

23 C. Tan, M. J. Selig, M. C. Lee and A. Abbaspourrad, Food Hydrocolloids, 2018, 84, 200-209.

24 P. Franco, E. Reverchon and I. De Marco, J. Supercrit. Fluids, 2019, 145, 31-38.

25 J. Zhang, J. Yu, Y. Zhang, Q. Li and J. R. Gong, Nano Lett., 2011, 11, 4774-4779.

26 L. Zhang, Y. Wang, N. Tang, P. Cheng, J. Xiang, W. Du and X. Wang, React. Funct. Polym., 2016, 99, 73-79.

27 I. M. Martins, S. N. Rodrigues, M. F. Barreiro and A. E. Rodrigues, Ind. Eng. Chem. Res., 2011, 50, 898-904.

28 I. Martins, S. N. Rodrigues, M. Barreiro and A. Rodrigues, J. Microencapsulation, 2009, 26, 667-675.

29 A. C. G. Moreira, I. M. Martins, I. Fernandes, M. F. Barreiro and A. E. Rodrigues, Can. J. Chem. Eng., 2016, 94, 469-475.

30 D. Silvestri, J. Mikšíček, S. Wacławek, R. Torres-Mendieta, V. V. T. Padil and M. Černík, Int. J. Biol. Macromol., 2019, 124, 396-402.

31 B. Hu, L. Han, H. Kong, K. Nishinari, G. O. Phillips, J. Yang and Y. Fang, Food Hydrocolloids, 2019, 88, 43-49.

32 M. Atgié, J. C. Garrigues, A. Chennevière, O. Masbernat and K. Roger, Food Hydrocolloids, 2019, 91, 319-330.
33 T. Zeng, Z. Wu, J. Zhu, S. Yin, C. Tang, L. Wu and X. Yang, Food Chem., 2017, 231, 122-130.

34 O. D. Orodu, K. B. Orodu, R. O. Afolabi and E. A. Dafe, Data in Brief, 2018, 19, 1773-1778.

35 G. E. Viacava, J. F. Ayala-Zavala, G. A. González-Aguilar and M. R. Ansorena, Postharvest Biol. Technol., 2018, 145, 125133.

36 Y. Wang, J. Jia, Y. Tian, X. Shu, X. Ren, Y. Guan and Z. Yan, LWT-Food Sci. Technol., 2018, 89, 604-609.

37 L. Lin, Y. Zhu, B. Thangaraj, M. A. S. Abdel-Samie and H. Cui, Carbohydr. Polym., 2018, 188, 243-251.

38 M. Ahmad, B. Ashraf, A. Gani and A. Gani, Int. J. Biol. Macromol., 2018, 109, 435-442.

39 Y. Wang, J. Shao, Z. Wang and Z. Lu, Food Res. Int., 2012, 49, 641-647.

40 S. Banerjee, P. Chattopadhyay, A. Ghosh, D. Goyary, S. Karmakar and V. Veer, Carbohydr. Polym., 2013, 93, 691697.

41 C. Dima, L. Pătraşcu, A. Cantaragiu, P. Alexe and Ş. Dima, Food Chem., 2016, 195, 39-48.

42 S. S. Gupta, S. Ghosh, P. Maiti and M. Ghosh, Food Sci. Technol. Int., 2012, 18, 549-558.

43 R. Chanamai and D. J. McClements, J. Food Sci., 2002, 67, 120-125.

44 D. Li, H. Wu, W. Huang, L. Guo and H. Dou, Eur. J. Lipid Sci. Technol., 2018, 120, 1700521.

45 S. Amiri and A. Rahimi, J. Ind. Text., 2018, 2025419403.

46 K. Munhuweyi, O. J. Caleb, A. J. van Reenen and U. L. Opara, LWT-Food Sci. Technol., 2018, 87, 413-422.

47 K. I. Matshetshe, S. Parani, S. M. Manki and O. S. Oluwafemi, Int. J. Biol. Macromol., 2018, 118, 676-682.

48 S. C. Chew, C. P. Tan and K. L. Nyam, J. Food Eng., 2018, 237, 78-85.

49 L. Hu, J. Zhang, Q. Hu, N. Gao, S. Wang, Y. Sun and X. Yang, J. Drug Delivery Sci. Technol., 2016, 36, 46-54.

50 N. Barbieri, A. Sanchez-Contreras, A. Canto, J. V. CauichRodriguez, R. Vargas-Coronado and L. M. Calvo-Irabien, Ind. Crops Prod., 2018, 121, 114-123.

51 K. I. Matshetshe, S. Parani, S. M. Manki and O. S. Oluwafemi, Int. J. Biol. Macromol., 2018, 118, 676-682.

52 R. Vilaplana, L. Pazmiño and S. Valencia-Chamorro, Postharvest Biol. Technol., 2018, 138, 56-63.

53 C. L. Chu, W. T. Liu, T. Zhou and R. Tsao, Can. J. Plant Sci., 1999, 79, 683-689.

54 R. K. Dubey, R. Kumar, J. P. N. Chansouria and N. K. Dubey, J. Food Saf., 2008, 28, 400-412.

55 N. Zhang, W. Guan and R. Yan, Sci. Technol. Food Ind., 2011, 32, 349-353.

56 M. B. Meah, R. A. Plumbley and M. J. Jeger, Mycol. Res., 1991, 95, 405-408.

57 D. Aswini, K. Prabakar, L. Rajendran, G. Karthikeyan and T. Raguchander, World J. Microbiol. Biotechnol., 2010, 26, 1107-1116. 